\title{
Transvenous extraction of His bundle pacing lead: New challenge in the field of lead extraction
}

\author{
Krzysztof Boczar ${ }^{1}$, Andrzej Ząbek ${ }^{1}$, Maciej Dębski ${ }^{1}$, \\ Jacek Gajek ${ }^{2}$, Jacek Lelakowski ${ }^{1,3}$, Barbara Małecka ${ }^{1,3}$ \\ ${ }^{1}$ Department of Electrocardiology, John Paul II Hospital, Krakow, Poland \\ ${ }^{2}$ Department of Cardiology, Wroclaw Medical University, Wroclaw, Poland \\ ${ }^{3}$ Institute of Cardiology, Jagiellonian University Medical College, Krakow, Poland
}

A 66-year-old male with ischemic heart disease, hypertrophic cardiomyopathy, left ventricular systolic dysfunction with ejection fraction of $40 \%$, chronic heart failure with frequent exacerbations and permanent atrial fibrillation was considered for implantation of implantable cardioverter-defibrillator with His bundle pacing (ICD-DR + HBP). The procedure was undertaken 11 months ago and following failed His bundle pacing implantation a dualsite right ventricular pacing was undertaken. The aim of the index hospital admission was to remove the present pacing system and establish His bundle pacing with biventricular pacing and defibrillator (CRT-D + HBP). Present ICD-DR system consisted of defibrillator lead and lumenless lead designed for His bundle pacing fixed in the intraventricular septum (Fig. 1A). During transvenous lead extraction, contrast venography of the ipsilateral arm venous confluence revealed complete occlusion of the left subclavian vein. In order to overcome this obstruction and regain venous access it was decided to target the removal of the lumenless lead. Given the relatively short lead dwell time, its position was secured by gripping the lead in the right atrium with a Needle Eye Snare, introduced via the femoral approach (Fig. 1B). Then, the lead was successfully and safely extracted via the access site with Byrd dilators (COOK MEDICAL LLC Bloomington, USA). Finally, the ICD lead was extracted and a new CRT-D + HBP system was implanted (Fig. 1C). The procedure was uneventful.

According to available literature, the present case is the first reported description for extraction of a lumenless lead dedicated for His bundle pacing facilitated by stabilization from the femoral approach to overcome complete venous occlusion.

Conflict of interest: None declared

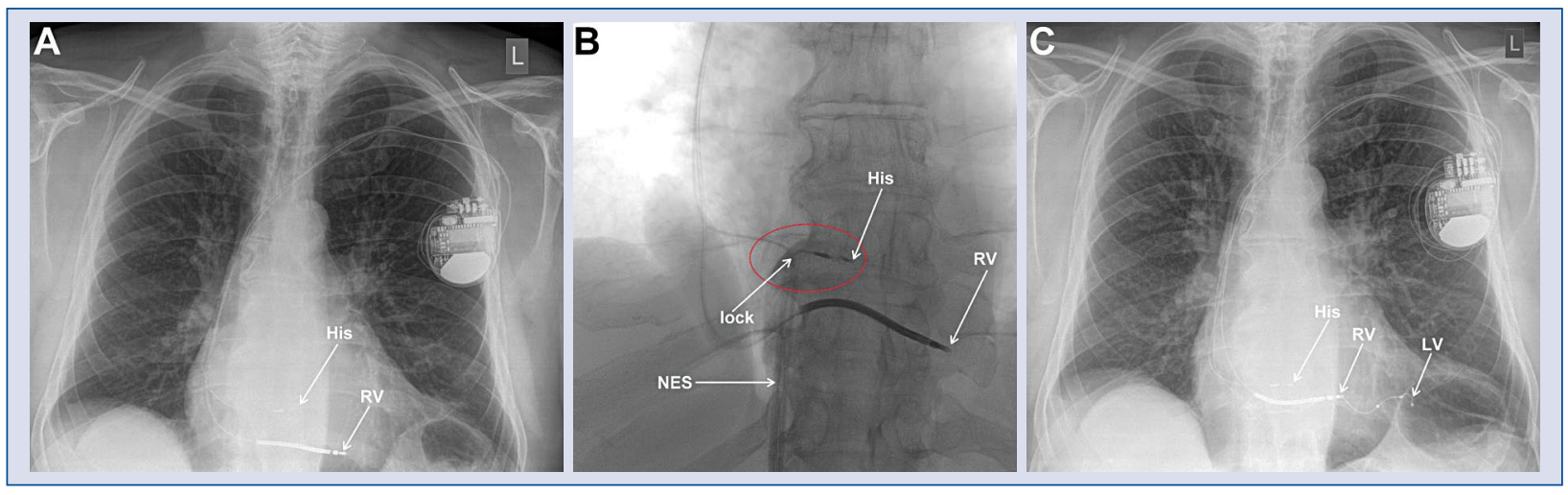

Figure 1. A. Initial ICD-DR system consisted of defibrillator lead Medtronic Sprint 6935M62 (RV) and Medtronic 3830-74cm lumenless lead designed for His bundle pacing (His) connected to atrial channel and fixed in intraventricular septum; B. Medtronic 3830-74cm lumenless lead (His) grasped (lock) in the right atrium with Needle Eye Snare (NES), (COOK MEDICAL LLC Bloomington, USA) introduced via femoral approach; C. The new CRT-D + HBP system at discharge; LV - left ventricular lead.

Address for correspondence: Krzysztof Boczar, MD, PhD, Department of Electrocardiology, John Paul II Hospital, ul. Prądnicka 80, 31-202 Kraków, Poland, tel:+48 12614 23 81, fax:+48 12633 23 99, e-mail:krzysiek.boczar@gmail.com 